\title{
CM-Biquad Filter Using Single DO-VDBA
}

\section{Chandra Ketu Yadav, Dinesh Prasad*, Zainab Haseeb, Laxya, Mayank Kumar}

Department of Electronics and Communication Engineering, Faculty of Engineering and Technology, Jamia Millia Islamia, New Delhi, India

Email: *dprasad@jmi.ac.in

How to cite this paper: Yadav, C.K., Prasad, D., Haseeb, Z., Laxya and Kumar, M. (2018) CM-Biquad Filter Using Single DO-VDBA. Circuits and Systems, 9, 133-139. https://doi.org/10.4236/cs.2018.99014

Received: August 21, 2018

Accepted: September 14, 2018

Published: September 17, 2018

Copyright (c) 2018 by authors and Scientific Research Publishing Inc. This work is licensed under the Creative Commons Attribution International License (CC BY 4.0).

http://creativecommons.org/licenses/by/4.0/

\section{(c) (i) Open Access}

\begin{abstract}
In this manuscript we present a current mode biquad using one dual output-voltage differencing buffered amplifier (DO-VDBA) and four passive components ( 2 grounded capacitors and 2 resistors). The proposed circuit offers very low active and passive sensitivity. The filter presented here is electronically tunable, frequency of oscillation (FO) can be tuned by controlling transconductance $\left(\mathrm{g}_{\mathrm{m}}\right)$ by varying the bias current $\left(\mathrm{I}_{\mathrm{B}}\right)$ of the circuit. The workability of proposed circuit is tested using PSPICE with $180 \mathrm{~nm}$ TSMC CMOS process parameters.
\end{abstract}

\section{Keywords}

Current Mode, Biquad Filter, Dual Output-Voltage Differencing Buffered Amplifier

\section{Introduction}

Biquads are the major components in the area of electronics. It is extensively used in numerous electronic applications, which include analog and digital signal processing, communication etc. [1]. Looking at the vast literature available, we are well aware that innumerable filters have been designed till date using several active building blocks and each of them has some advantages and disadvantages too. Current mode filters have proven its vitality in several aspects, some of them are high performance, lower power consumption, miniaturization of circuit, higher frequency range, increased slew rate and linearity. So in this paper the current mode is exploited to get such benefits. Filters available in literature have also reportedly suffered few problems such as smaller frequency range, lack of tunability and excessive use of passive components.

The filter proposed in this paper uses current mode VDBA, it is single input and dual output and it is electronically tunable as well [2].

FB-VDBA is well known for voltage-mode analog signal processing. It is a 
fully-differential structure. It is used to realize floating inductor [3].

Using voltage mode configuration, biquad filter has also been realized using VDBA. Those filters use 2 active elements and $2 / 3$ passive components. They were having low passive sensitivity and acceptable value of THD [4].

An extremely convenient multiphase oscillator with reduced complexity as an easy non-tunable replacement to classical conceptions employing lossy integrators in phase-shifted loop has been designed using VDBA/VDIBA. Linearly tunable quadrature oscillator and square wave generator were also reported in the literature [5].

Single VDIBA and a capacitor, can be used to realize a novel voltage-mode (VM) resistorless, 1st-order all-pass filter (APF) [6].

Several analog signal-processing filters are also available using current mode circuit; CDBA is one such ABB [7].

$\mathrm{CM}$ filters have proven its vitality in several aspects, some of them are high performance, lower power consumption, miniaturization of circuit, higher frequency range, increased slew rate and linearity, so in this manuscript the CM is exploited to get such benefits. Filters available in literature have also reportedly suffered few problems such as smaller frequency range, lack of tunability and excessive use of passive components. FB-VDBA is very useful building block for analog circuit design. It is also helpful in the designing of circuit with least number of passive components [8].

Voltage mode configuration has also been proved helpful in design of a current-mode and voltage-mode electronically tunable quadrature oscillator that consists of both voltage and current output [9].

To the best awareness of the authors no same type of current mode biquad is available in the open literature. So, in this manuscript we have proposed a biquad using single DO-VDBA whose frequency is electronically controllable. Offering very low active and passive sensitivities are also important characteristics of this circuit.

\section{The Proposed New Structure}

The DO-VDBA is represented in its symbolic form as shown in Figure 1, where the input terminals are given by $P$ and $N$ while the output terminals are denoted by $Z, W^{+}$and $W^{-}$. Current flowing through " $Z$ " terminal is given by the difference of $V_{P}$ and $V_{N}$ by transconductance $\left(g_{m}\right)$. The $V_{W}$ is same as the $V_{Z}$. The electronic controllability of DO-VDBA has advantages over the traditional VDBA by possibility of controlling of $g_{m}$ through the bias current $I_{B}$.

The DO-VDBA can be given by the following matrix:

$$
\left[\begin{array}{c}
I_{p} \\
I_{n} \\
I_{z} \\
V_{w^{+}} \\
V_{w^{-}}
\end{array}\right]=\left[\begin{array}{ccc}
0 & 0 & 0 \\
0 & 0 & 0 \\
g_{m} & -g_{m} & 0 \\
0 & 0 & 1 \\
0 & 0 & -1
\end{array}\right]\left[\begin{array}{l}
V_{p} \\
V_{n} \\
V_{z}
\end{array}\right]
$$




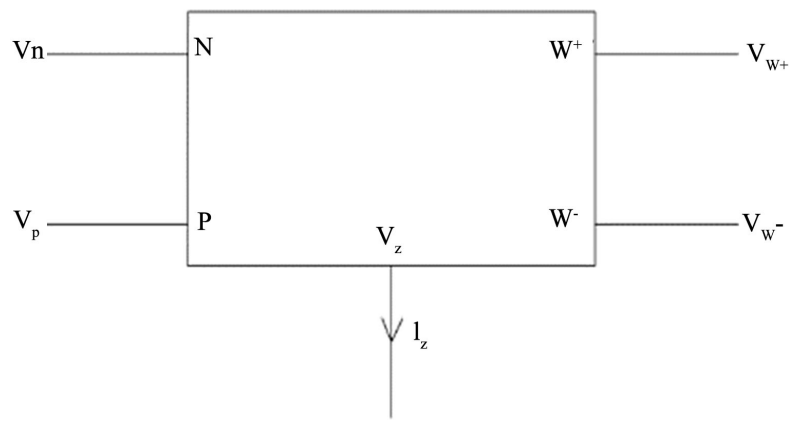

Figure 1. Symbolic notation of the DO-VDBA.

After applying KCL at different nodes of Figure 2 the proposed structure yields the following current transfer functions:

$$
\begin{aligned}
& T_{1}(s)=\left.\frac{I_{C 1}}{I_{i n}}\right|_{H P F}=\frac{s^{2}}{s^{2}+\frac{s}{R_{1} C_{1}}+\frac{g_{m}}{R_{1} C_{1} C_{2}}} \\
& T_{2}(s)=\left.\frac{I_{C 2}}{I_{\text {in }}}\right|_{B P F}=\frac{-\left(\frac{s g_{m}}{C_{1}}\right)}{s^{2}+\frac{s}{R_{1} C_{1}}+\frac{g_{m}}{R_{1} C_{1} C_{2}}} \\
& T_{3}(s)=\left.\frac{I_{R 2}}{I_{\text {in }}}\right|_{L P F}=\frac{\left(\frac{g_{m}}{R_{2} C_{1} C_{2}}\right)}{s^{2}+\frac{s}{R_{1} C_{1}}+\frac{g_{m}}{R_{1} C_{1} C_{2}}} \\
& T_{4}(s)=\left.\frac{I_{4}}{I_{i n}}\right|_{A P F}=\frac{s^{2}-\frac{s g_{m}}{C_{1}}+\frac{g_{m}}{R_{2} C_{1} C_{2}}}{s^{2}+\frac{s}{R_{1} C_{1}}+\frac{g_{m}}{R_{1} C_{1} C_{2}}}
\end{aligned}
$$

where $I_{4}=I_{c 1}+I_{c 2}+I_{r 2}$

$$
T_{5}(s)=\left.\frac{I_{5}}{I_{i n}}\right|_{B R F}=\frac{s^{2}+\frac{g_{m}}{R_{2} C_{1} C_{2}}}{s^{2}+\frac{s}{R_{1} C_{1}}+\frac{g_{m}}{R_{1} C_{1} C_{2}}}
$$

where $I_{5}=I_{c 1}+I_{r 2}$.

The cutoff frequency $\omega_{0}$, bandwidth $(B W)$ and quality factor $Q_{0}$ are given by:

$$
\begin{gathered}
\omega_{0}=\sqrt{\frac{g_{m}}{R_{1} C_{1} C_{2}}} \\
B W=\frac{\omega_{0}}{Q_{0}}=\frac{1}{R_{1} C_{1}} \\
Q_{0}=\sqrt{\frac{g_{m} R_{1} C_{1}}{C_{2}}}
\end{gathered}
$$




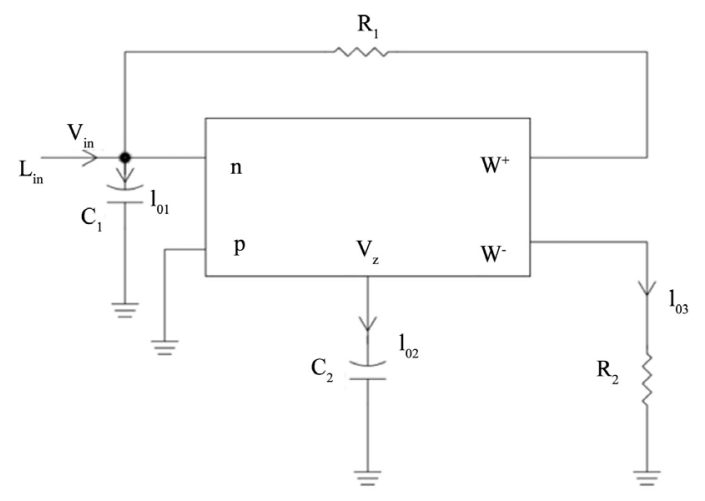

Figure 2. The proposed configuration.

\section{Sensitivity Analysis}

The various sensitivities of $\omega_{0}$ and $Q_{0}$ w.r.t. each passive and active element are:

Sensitivity of $y$ with respect to $x$ is symbolized as

$$
S_{x}^{y}=\frac{\partial y / y}{\partial x / x}=\frac{x}{y} \frac{\partial y}{\partial x}
$$

It's active and passive sensitivity of circuit analysis parameter are expressed as:

1)

$$
\begin{aligned}
S_{g_{m}}^{\omega_{0}} & =\frac{\partial \omega_{0} / \omega_{0}}{\partial g_{m} / g_{m}}=\frac{g_{m}}{\omega_{0}} \frac{\partial \omega_{0}}{\partial g_{m}}=\frac{g_{m} \sqrt{R_{1} C_{1} C_{2}}}{\sqrt{g_{m}}} \frac{\partial}{\partial g_{m}} \sqrt{\frac{g_{m}}{R_{1} C_{1} C_{2}}} \\
& =\frac{1}{2} \frac{g_{m} \sqrt{R_{1} C_{1} C_{2}}}{\sqrt{g_{m}}} \frac{1}{\sqrt{R_{1} C_{1} C_{2}}} \frac{1}{\sqrt{g_{m}}}=\frac{1}{2}
\end{aligned}
$$$$
S_{C_{2}}^{Q_{0}}=\frac{\partial Q_{0} / Q_{0}}{\partial C_{2} / C_{2}}=\frac{C_{2}}{Q_{0}} \frac{\partial Q_{0}}{\partial C_{2}}=\frac{C_{2} \sqrt{C_{2}}}{\sqrt{g_{m} R_{1} C_{1}}} \frac{\partial}{\partial C_{2}} \sqrt{\frac{g_{m} R_{1} C_{1}}{C_{2}}}
$$

2)

$$
=\frac{-1}{2} \frac{C_{2} \sqrt{C_{2}} \sqrt{g_{m} R_{1} C_{1}}}{\sqrt{g_{m} R_{1} C_{1}}} \frac{1}{\sqrt[3]{C_{2}}}=\frac{-1}{2}
$$

3)

$$
S_{R_{1}}^{\omega_{0}}=\frac{\partial \omega_{0} / \omega_{0}}{\partial R_{1} / R_{1}}=\frac{R_{1}}{\omega_{0}} \frac{\partial \omega_{0}}{\partial R_{1}}=\frac{R_{1} \sqrt{R_{1} C_{1} C_{2}}}{\sqrt{g_{m}}} \frac{\partial}{\partial R_{1}} \sqrt{\frac{g_{m}}{R_{1} C_{1} C_{2}}}
$$

$$
=\frac{-1}{2} \frac{R_{1} \sqrt{R_{1} C_{1} C_{2}}}{\sqrt{g_{m}}} \frac{\sqrt{g_{m}}}{\sqrt{C_{1} C_{2}}} \frac{1}{\sqrt[3]{R_{1}}}=\frac{-1}{2}
$$

4)

$$
S_{C_{1}}^{\omega_{0}}=\frac{\partial \omega_{0} / \omega_{0}}{\partial C_{1} / C_{1}}=\frac{C_{1}}{\omega_{0}} \frac{\partial \omega_{0}}{\partial C_{1}}=\frac{C_{1} \sqrt{R_{1} C_{1} C_{2}}}{\sqrt{g_{m}}} \frac{\partial}{\partial C_{1}} \sqrt{\frac{g_{m}}{R_{1} C_{1} C_{2}}}
$$

$$
=\frac{-1}{2} \frac{C_{1} \sqrt{R_{1} C_{1} C_{2}}}{\sqrt{g_{m}}} \frac{\sqrt{g_{m}}}{\sqrt{R_{1} C_{2}}} \frac{1}{\sqrt[3]{C_{1}}}=\frac{-1}{2}
$$

$$
S_{C_{2}}^{\omega_{0}}=\frac{\partial \omega_{0} / \omega_{0}}{\partial C_{2} / C_{2}}=\frac{C_{2}}{\omega_{0}} \frac{\partial \omega_{0}}{\partial C_{2}}=\frac{C_{2} \sqrt{R_{1} C_{1} C_{2}}}{\sqrt{g_{m}}} \frac{\partial}{\partial C_{2}} \sqrt{\frac{g_{m}}{R_{1} C_{1} C_{2}}}
$$

5)

$$
=\frac{-1}{2} \frac{C_{2} \sqrt{R_{1} C_{1} C_{2}}}{\sqrt{g_{m}}} \sqrt{\frac{g_{m}}{R_{1} C_{1}}} \frac{1}{\sqrt[3]{C_{2}}}=\frac{-1}{2}
$$




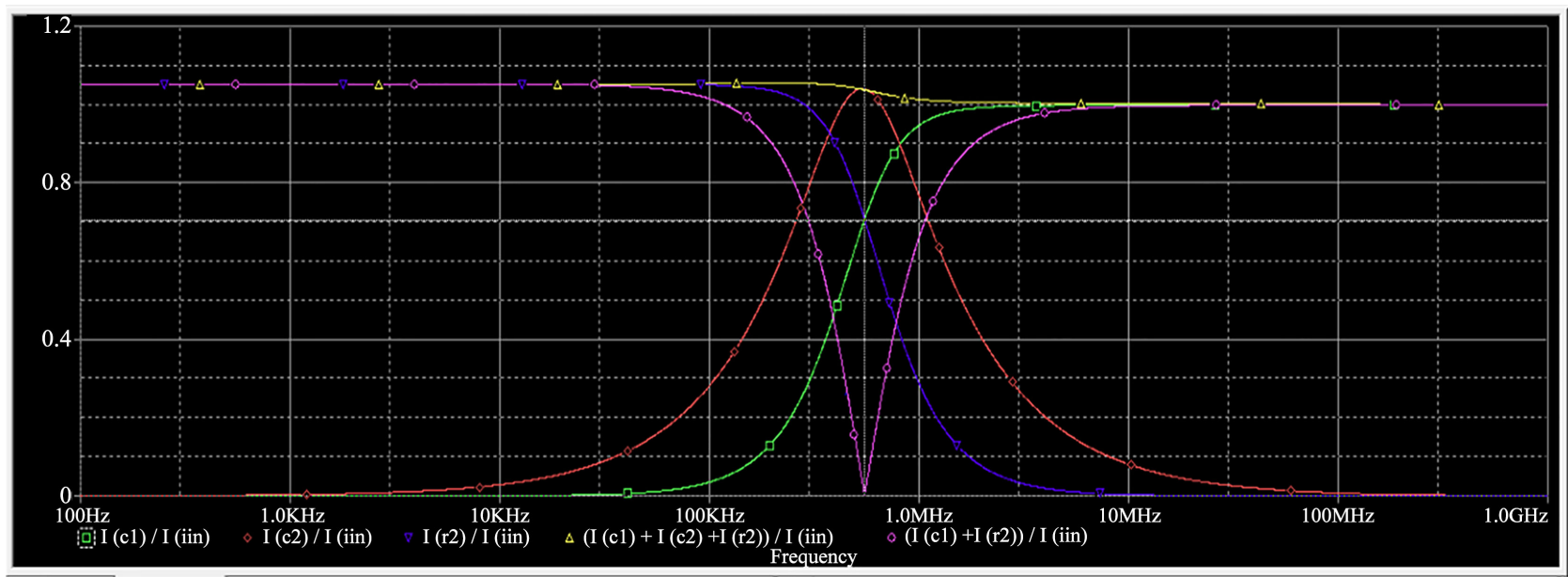

Figure 3. Frequency response of the proposed biquad.

6)

$$
S_{g_{m}}^{Q_{0}}=\frac{\partial Q_{0} / Q_{0}}{\partial g_{m} / g_{m}}=\frac{g_{m}}{Q_{0}} \frac{\partial Q_{0}}{\partial g_{m}}=\frac{g_{m} \sqrt{C_{2}}}{\sqrt{g_{m} R_{1} C_{1}}} \frac{\partial}{\partial g_{m}} \sqrt{\frac{g_{m} R_{1} C_{1}}{C_{2}}}
$$

$$
\begin{aligned}
& =\frac{1}{2} \frac{g_{m} \sqrt{C_{2}}}{\sqrt{g_{m} R_{1} C_{1}}} \sqrt{\frac{R_{1} C_{1}}{C_{2}}} \frac{1}{\sqrt{g_{m}}}=\frac{1}{2} \\
& S_{R_{1}}^{Q_{0}}=\frac{\partial Q_{0} / Q_{0}}{\partial R_{1} / R_{1}}=\frac{R_{1}}{Q_{0}} \frac{\partial Q_{0}}{\partial R_{1}}=\frac{R_{1} \sqrt{C_{2}}}{\sqrt{g_{m} R_{1} C_{1}}} \frac{\partial}{\partial R_{1}} \sqrt{\frac{g_{m} R_{1} C_{1}}{C_{2}}}
\end{aligned}
$$

7)

$$
=\frac{1}{2} \frac{R_{1} \sqrt{C_{2}}}{\sqrt{g_{m} R_{1} C_{1}}} \sqrt{\frac{g_{m} C_{1}}{C_{2}}} \frac{1}{\sqrt{R_{1}}}=\frac{1}{2}
$$

$$
S_{C_{1}}^{Q_{0}}=\frac{\partial Q_{0} / Q_{0}}{\partial C_{1} / C_{1}}=\frac{C_{1}}{Q_{0}} \frac{\partial Q_{0}}{\partial C_{1}}=\frac{C_{1} \sqrt{C_{2}}}{\sqrt{g_{m} R_{1} C_{1}}} \frac{\partial}{\partial C_{1}} \sqrt{\frac{g_{m} R_{1} C_{1}}{C_{2}}}
$$

$$
=\frac{1}{2} \frac{C_{1} \sqrt{C_{2}}}{\sqrt{g_{m} R_{1} C_{1}}} \sqrt{\frac{g_{m} R_{1}}{C_{2}}} \frac{1}{\sqrt{C_{1}}}=\frac{1}{2}
$$

From above values of sensitivity, concluding the values here:

$$
S_{g_{m}}^{\omega_{0}}=\frac{1}{2}, S_{R_{1}, C_{1}, C_{2}}^{\omega_{0}}=-\frac{1}{2}, S_{g_{m}, R_{1}, C_{1}}^{Q_{0}}=\frac{1}{2}, S_{C_{2}}^{Q_{0}}=-\frac{1}{2}
$$

From Equation (10) it is clear that the proposed circuit offers small active and passive sensitivities.

\section{Simulation Result}

To show the functionality the presented biquad is tested using SPICE simulations. For this purpose we use CMOS DO-VDBA [5] with power supply voltage taken as $V_{D D}=-V_{S S}=1.2 \mathrm{~V}, I_{b}=50 \mu \mathrm{A}$ biasing current and passive elements with $C_{1}=0.1 \mathrm{nF}, C_{2}=0.2 \mathrm{nF}, R_{1}=2 \mathrm{k} \Omega$ and $R_{2}=2 \mathrm{k} \Omega$. The frequency response of the circuit is depicted in Figure 3 with the cutoff frequency $f_{0}=0.78 \mathrm{MHz}$.

\section{Conclusion}

In this manuscript we present an application of dual output voltage differencing 
buffered amplifier as current mode biquad filter using single DOVDBA and 4 passive components ( 2 resistors and 2 grounded capacitors as required for IC fabrication). The presented circuit offers low active and passive sensitivity. The FO can be tuned electronically by varying transconductance $\left(g_{m}\right)$ of circuit. The active and passive sensitivity of the proposed work are not more than one. It means that it has low sensitivity. The practicability of reported circuit is tested using pSPICE simulation with $180 \mathrm{~nm}$ process parameters of TSMC CMOS.

\section{Acknowledgements}

This research is supported by the "Young Faculty Research Fellowship and Research/Contingency Grant", under the "Visvesvaraya PhD Scheme for Electronics and IT", Ministry of Electronics and Information Technology, Govt. of India.

\section{Conflicts of Interest}

The authors declare no conflicts of interest regarding the publication of this paper.

\section{References}

[1] Toumazou, C., Lidgey, F.J. and Haigh, D.G. (1992) Analogue IC Design: The Current Mode Approach. IEE Circuits and Systems Series: Institution of Electrical Engineers. IET, Stevenage.

[2] Biolek, D., biolkova, V. and Kolka, Z. (2010) All Pass Filter Employing Fully Balanced Voltage Differencing Buffered Amplifier. Proceeding of IEEE Latin American Symposium Circuits and Systems, Foz do Iguacu, 24-26 February 2010, 232-235.

[3] Biolkova, V., Kolka, Z. and Biolek, D. (2009) Fully Balanced Voltage Differencing Buffered Amplifier and Its Application. 2009 52nd IEEE International Midwest Symposium on Circuits and System, Cancun, 2-5 August 2009, 45-48. https://doi.org/10.1109/MWSCAS.2009.5236157

[4] Kacar, F., Yesil, A. and Noori, A. (2012) New CMOS Realization of Voltage Differencing Buffered Amplifier and Its Biquad Filter Applications. Radio Engineering, 21, 333-339.

[5] Sotner, R., Jerabek, J. and Herencsar, N. (2013) Voltage Differencing Buffered/Inverted Amplifiers and Their Applications for Signal Generation. Radio Engineering, 22, 490-504.

[6] Acar, C. and Ozogue, S. (1999) A New Versatile Building Block: Current Differencing Buffered Amplifier. Microelectronics Journal, 30, 157-160. https://doi.org/10.1016/S0026-2692(98)00102-5

[7] Herencsar, N., Minaei, S., Koton, J., Yuce, E. and Vrba, K. (2013) New Realization of Dual-Output Voltage Mode All Pass Filtering Using Voltage Differencing Invereted Buffered Amplifier. Analog Integrated Circuits and Signal Processing, 74, 141-154. https://doi.org/10.1007/s10470-012-9936-2

[8] Yesil, A., Kacar, F. and Gurkan, K. (2016) Design and Experimental Evolution of Quadrature Oscillator Employing Single Fully Balanced Voltage Differencing Buffered Amplifier. Journal of electrical Engineering, 67, 137-142. 
https://doi.org/10.1515/jee-2016-0019

[9] Yesil, A. and Kacar, F. (2018) Current and Voltage Mode Quadrature Oscillator Based on Voltage Differencing Buffered Amplifier. Electrical, 18, 6-12.

https://doi.org/10.5152/iujeee.2018.1802 